\title{
In Vitro Degradation of Poly(caprolactone)/nHA Composites
}

\author{
Esperanza Díaz, Iban Sandonis, and María Blanca Valle \\ Departamento de Ingeniería Minera, Metalúrgica y Ciencia de Materiales, Escuela Técnica Superior de Náutica y Máquinas Navales, \\ Universidad del País Vasco (UPV/EHU), María Díaz de Haro 68, 48920 Portugalete, Spain \\ Correspondence should be addressed to Esperanza Díaz; esperanza.diaz@ehu.es
}

Received 30 April 2014; Revised 11 September 2014; Accepted 15 September 2014; Published 30 October 2014

Academic Editor: Zhongkui Hong

Copyright (C) 2014 Esperanza Díaz et al. This is an open access article distributed under the Creative Commons Attribution License, which permits unrestricted use, distribution, and reproduction in any medium, provided the original work is properly cited.

The degradation behavior and mechanical properties of polycaprolactone/nanohydroxyapatite composite scaffolds are studied in phosphate buffered solution (PBS), at $37^{\circ} \mathrm{C}$, over 16 weeks. Under scanning electron microscopy (SEM), it was observed that the longer the porous scaffolds remained in the PBS, the more significant the thickening of the pore walls of the scaffold morphology was. A decrease in the compressive properties, such as the modulus and the strength of the PCL/nHA composite scaffolds, was observed as the degradation experiment progressed. Samples with high nHA concentrations degraded more significantly in comparison to those with a lower content. Pure PCL retained its mechanical properties comparatively well in the study over the period of degradation. After the twelfth week, the results obtained by GPC analysis indicated a significant reduction in their molecular weight. The addition of nHA particles to the scaffolds accelerated the weight loss of the composites and increased their capacity to absorb water during the initial degradation process. The addition of these particles also affected the degradation behavior of the composite scaffolds, although they were not effective at compensating the decrease in $\mathrm{pH}$ prompted by the degradation products of the PCL.

\section{Introduction}

The manufacture of appropriate scaffolds of the right shape, dimensions, and properties is of the utmost importance for tissue engineering. Specific applications may have particular requirements but, generally speaking, they should facilitate cell adhesion, differentiation, and proliferation. They should also guide tissue and organ creation, promoting survival and integration with native tissue and decomposing when no longer necessary. Scaffolds are therefore usually expected to be biocompatible, porous, three-dimensional, bioactive, biodegradable, and with similar mechanical properties to those of the replaced tissue [1]. A large array of biocompatible materials has been used for this purpose.

Polycaprolactone (PCL) is the most widely studied substance in the family of polylactones. Its glass transition temperature is of around $-60^{\circ} \mathrm{C}$, low melting temperature is of 58 to $63^{\circ} \mathrm{C}$, and exceptional blend-compatibility has stimulated extensive research into its potential biomedical applications [2]. This is prepared by ring-opening polymerization of the cycling monomer $\varepsilon$-caprolactone. Polycaprolactone is a semicrystalline linear resorbable aliphatic polyester, generally considered a nontoxic, tissue compatible polymer. It is subjected to biodegradation, because of the susceptibility of its aliphatic ester linkage to hydrolysis. The degradation products, caproic acid, are metabolized via the tricarboxylic acid cycle or eliminated by direct renal secretion [3]. PCL is therefore a biodegradable and bioresorbable polymeric material.

PCL has been ignored for most of two decades, partly because of its long-term degradation (up to 1 year) that is unsuitable for some applications $[3,4]$. However, its rheological and viscoelastic properties have recently revived interest in its properties. Numerous in vitro and in vivo studies were performed prior to FDA-approval of these products. Recently extensive investigations have examined its ability as a potential bone tissue engineering material, but PCL alone may not be a satisfactory material for the creation of bone substitutes $[4,5]$. In other studies, the polymeric scaffold was blended with bioceramic materials-silica and hydroxyapatite $-[6,7]$ in order to improve its bioactivity. The addition of these materials modifies its structure and therefore its properties. The same paper [7] also showed that the hydrolysis rate may be altered by the addition of hydroxyapatite nanoparticles. 
There are few results on the effects of nHA on PCL ageing and degradation behavior [8-10]. Many articles have studied PCL degradation, in relation to such aspects as $\mathrm{pH}$, crystallinity, and the $\%$ of absorbed water, among others [8].

The fabrication technique used in this paper was thermally induced phase separation (lyophilization), as well as the incorporation of $\mathrm{nHA}$ prior to the fabrication of the scaffold, a technique that permitted homogeneous dispersion of the nHA particles. In this paper, the effect of nHA content on the evolution and the degradability of the PCL/nHA composite scaffolds were studied. During the degradation, some properties of the polymer might change. However, the effect of such a change on the degradability of the composite scaffolds has yet to be fully elucidated. In this study, their degradation mechanism was discussed in detail by analyzing changes in $\mathrm{pH}$, weight loss, molecular weight, morphology, and mechanical properties.

\section{Materials and Methods}

2.1. Materials. Polycaprolactone was supplied by Purac Biomaterials Purasorb PCL 12 (Holland) [9]. The solvent, 1,4-dioxane (Panreac p.a., Barcelona, Spain), was distilled by conventional methods. The weight-average relative molecular weight $\mathrm{Mw}=130490, \mathrm{Mn}=79760$, and polydispersity $\mathrm{Mw} / \mathrm{Mn}=1.636$ of $\mathrm{PCL}$ were determined using gel permeation chromatography (GPC, Perkin Elmer 200) in tetrahydrofuran (THF). GPC was performed with a THF solvent using a Perkin Elmer 200 reflective index detector. Calibration was done in accordance with polystyrene standards with a flow rate of $1 \mathrm{~mL} / \mathrm{min}$. Nanohydroxyapatite (nHA) was supplied by Aldrich Chemistry (USA), with a particle size > $200 \mathrm{~nm}$ and $\mathrm{Mw}=502.31 \mathrm{~g} / \mathrm{mL}$. Phosphate buffer solution in water (PBS), supplied by Fluka Analytical (Sigma Aldrich, USA) at a $\mathrm{pH}$ of 7.2, was used as the degradation fluid.

\subsection{Fabrication of Porous Scaffolds. PCL and PCL/nHA} composite scaffolds were synthesized according to a protocol described in our previous reference [9]. In brief, PCL was dissolved in 1,4 -dioxane $(2.5 \%(\mathrm{w} / \mathrm{v}))$. Then, nHA particles were homogeneously dispersed in the above suspension by ultrasonic stirring. Highly porous composite scaffolds with different nHA proportions of total polymer mass $(0 \%, 10 \%$, $30 \%$, and $50 \%$ ) were obtained after freezing and freeze-drying the resultant solutions for several days. Porous foam scaffolds with porosities of up to $90 \%$ were obtained by this method.

2.3. In Vitro Degradation. In preparation for the degradation experiment, the samples were finely cut into $15 \mathrm{~mm}$ diameter disks, weighed, and totally immersed in glass test tubes filled with $10 \mathrm{~mL}$ of PBS, where they were evenly incubated in a thermostated oven at $37^{\circ} \mathrm{C}$. The specimens were recovered after $1,2,4,6,8,12$, and 16 weeks under the same conditions, carefully wiped, and then weighed to determine water absorption. A pH meter PCE 228 by PCE Instruments (Spain) was used to determine the $\mathrm{pH}$ alteration in the medium. Eventually, the degraded samples were dried over 2 additional weeks to a constant weight.
Water absorption and weight loss were evaluated by weighing, taking into account the original weight of each sample $\left(W_{0}\right)$ and the residual weight, after degradation of the same specimen $\left(W_{r}\right)$ that had completely dried. The water absorption percentage, $W_{a} \%$, was calculated by the following equation:

$$
W_{a} \%=\frac{W_{w}-W_{r}}{W_{r}} \times 100,
$$

where $W_{w}$ is the weight of the wet/swallow specimen after removing surface water, and $W_{r}$ is the residual weight of a completely dry sample after degradation.

Weight loss percentage $\left(W_{L} \%\right)$ was estimated with the following equation:

$$
W_{L} \%=\frac{W_{0}-W_{r}}{W_{0}} \times 100 .
$$

2.4. Scaffold Porosity. The porosity of the scaffolds was quantified as in the previous reference $[11,12]$. Briefly, the bulk density of the polymer $\left(\rho_{a}\right)$ was calculated by mercury pycnometry measurements on each material. The percentage porosity $(\% P)$ was calculated by the following equation:

$$
\% P=\left(1-\frac{\rho_{a}}{\rho_{p}}\right) \times 100,
$$

where $\rho_{p}$ is the bulk density of the polymer.

2.5. SEM Analysis. The bulk morphology of the scaffolds was examined using scanning electron microscopy (SEM) (HITACHI S-3400N, Tokyo, Japan). Prior to analysis, the samples were coated with a layer of gold, in a JEL Ion Sputter JFC-1100 at $1200 \mathrm{~V}$ and $5 \mathrm{~mA}$, to avoid sample charging under the electron beam.

2.6. Mechanical Properties. Mechanical testing of the pore scaffolds was performed using a Universal Testing Machine (Instrom, Model: 4502, UK). The PLLA and PCL scaffolds preparation procedure was the same as described for the fabrication methods. The diameter of the scaffold disk was $11 \mathrm{~mm}$ and its thickness was $2 \mathrm{~mm}$. The compressive modulus was defined as the initial linear modulus and the yield strength was determined from the intersection of the two tangents on the stress-strain curve around the yield point. Four scaffolds were mechanically tested for each sample.

\section{Results and Discussion}

The kinetics of degradation are highly dependent upon polymeric molecular weight. Structures with a high molecular weight take much longer to degrade, as mediated through the chain length of the polymer. A higher molecular weight increases the chain length, requiring a greater number of ester bonds to be cleaved, in order to generate water-soluble monomers/oligomers so that erosion can proceed, although degradation consequently takes longer [13]. 
The polymer used was of a higher molecular weight $\mathrm{Mw}=$ 130490. PCL is a hydrophobic, semicrystalline polymer; its crystallinity tends to decrease with increasing molecular weight.

3.1. SEM. The particles, included before the lyophilization process, are found uniformly distributed in the polymer matrix that forms these porous substrates. Scaffold porosity exceeded $90 \%$. In previous publications, the research group $[11,12,14]$ has reported that, when using 1,4-dioxane and the resulting heat treatment in the manufacturing process, the pore structure and morphology were controlled by the solidliquid phase separation process of the polymer solution. The solid-liquid separation was attributed to the crystallization of the solvent.

As we can see in Figure 1, the presence of nHA particles in the PCL scaffolds does not appear to change their pore size. However, their presence does cause a more irregular morphology, as these particles perturb the crystallization of the solvent and change the patterns of crystal growth, forming more irregular crystals in the solvent. Both polymer and nHA particles were expelled from the crystallization front forming a rich phase and a continuous PCL/nHA skeleton, and the spaces formerly occupied by the solvent crystals became the pores of the scaffold, leaving behind the polymer as a foam. As a result of irregular solvent crystal growth, the pores and the structure became irregular (more isotropic). Samples with a low content of nHA had a more porous appearance. The addition of high amounts of nanoparticles modified the structure to give it a fibrous appearance, as seen in the micrographs of Figures 1(a), 1(b), 1(c), and 1(d).

Figures $1(\mathrm{e}), 1(\mathrm{f}), 1(\mathrm{~g})$, and $1(\mathrm{~h})$ show that the longer the porous scaffold supports remained in PBS, the more significant the thickening on the walls of the PCL and PCL/nHA $10 \mathrm{wt} \%$ pores was. For the rest of the scaffolds, which have a higher percentage of nanoparticles, the fibrous appearance may be appreciated when the pores are stretched. In the micrographs of these samples, neither did degradationrelated products appear nor were nHA particles exposed outside the scaffolds walls and the surface morphology cannot be seen to change from a smooth to an abrupt surface as other authors have observed in their work $[8,14,15]$.

3.2. Mechanical Properties. The reconstruction of a segmental defect in bone tissue is required to restore the structure and function of the affected site. The scaffold acts as a carrier of proteins and growth factors, while also supporting the load that the bone would normally sustain, until there is sufficient natural bone growth. That is why the analysis of the mechanical properties during the degradation process is an important part of our research.

In previous works $[11,12,14]$, a great variety of scaffolds of PLLA, PCL, and DLGA have been studied, in which the mechanical properties were dependent on the pore structure and pore wall morphology. The mechanical properties such as compressive modulus and yield strength as a function of degradation time can be observed in Figures 2 and 3. These mechanical properties (Figures 2(a) and 2(b)) increased linearly with the nHA particles added to the scaffolds, except for the sample of more content in nHA that remained constant. This change might be because of the sudden shift in the morphology of the scaffold, which assumed a fibrous appearance due to high concentrations of the nanoparticles. The influence of the nHA particles is important, despite their nanometric scale and uniform distribution.

In Figures 3(a) and 3(b), we can see the compressive modulus and yield strength as a function of degradation time. These mechanical properties decreased continuously and slowly as from the first week, during the degradation time. The samples with a higher nHA wt $\%$ had reached low values after 12 weeks. While the pure PCL samples were found to retain their mechanical properties over time, the degradation process was most drastically affected by the samples containing nHA. This behavior is similar to the findings of this research group in connection with PLLA/nHA and other studies $[11,12,14]$. As from week 12 , random hydrolytic chain scission took place and produced an overall reduction in molecular weight. The hydrolytic chain cleavage of PCL is in its amorphous regions, as those regions are more susceptible to the hydrolytic attack than densely packed crystalline regions. If water molecules can diffuse into the polymer bulk and hydrolyse the chains enabling the monomers or oligomers to diffuse out, erosion will occur gradually and equilibrium for this diffusion-reaction phenomenon would be achieved. If this equilibrium is disturbed, the degradation mechanism could provoke internal autocatalysis, via the carboxyl and hydroxyl end group by-products. The study of the PCL scaffolds detected no evidence of internal catalysis over the period of degradation, as the uniform molecular weight distribution over time and cross-sectional examination of the scaffold struts was able to show over the 16 weeks of the study.

The mechanical properties obtained are not comparable to those obtained by other authors $[9,10]$. This is surely due to the different manufacturing technique employed, which causes a different scaffold morphology and porosity.

3.3. Weight Loss \%. Figure 4 shows the weight loss of the PCL and the PCL/nHA composite scaffolds against incubation time in PBS solution $(\mathrm{pH}=7.2)$ at $37^{\circ} \mathrm{C}$. Generally, degradation rates of the polymer were affected by their structure, molecular weight, and other structural characteristics. PCL is hydrophobic and has a highly crystalline structure that does not allow fast water penetration into the PCL bulk. Though the mechanism of PCL degradation is known [5] as random hydrolytic chain scission of the ester linkage, the degradation kinetics were relatively slower than other degradable polyesters such as PLLA or DLGA $[12,14]$. In Figure 4 , we can see how, at the end of week 16 , weight loss is very small $-0.2 \%$, for the PCL sample-however, the samples with high nHA content experienced greater weight loss. These results indicate that samples with nHA lose a little more weight over the same period of degradation. It could be that the nHA will support or give off porous nanoparticles that might actually increase the rate of degradation. The latter should be consistent with the values obtained by GPC (Figure 5). 


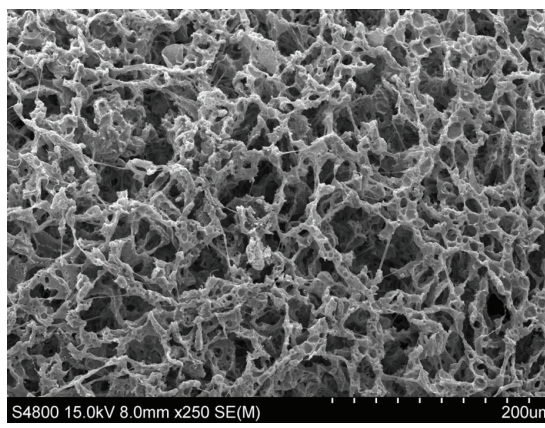

(a)

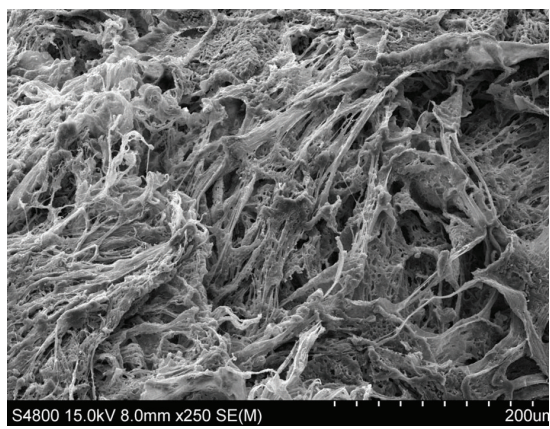

(d)

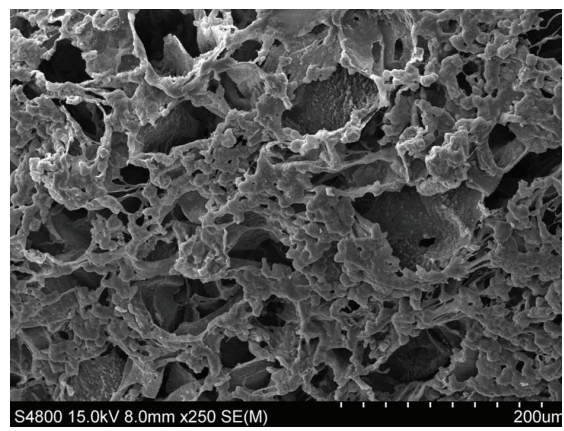

(g)

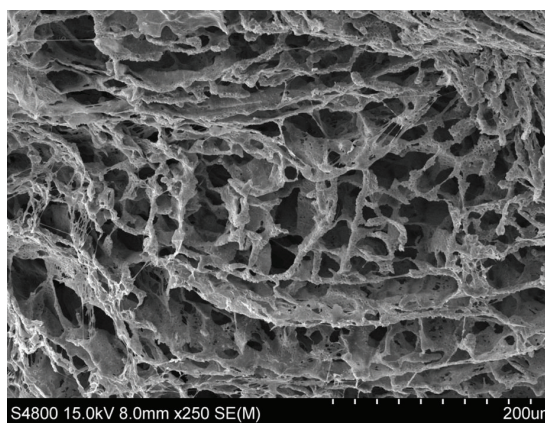

(j)

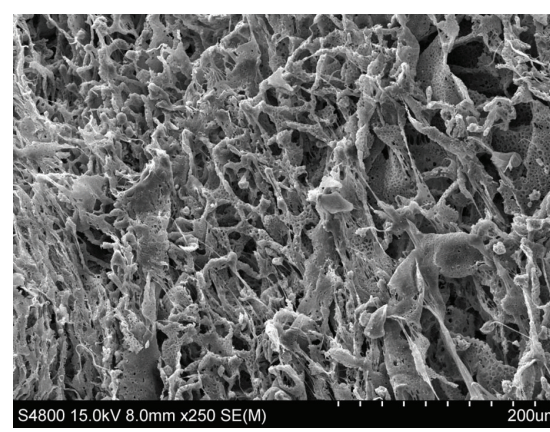

(b)

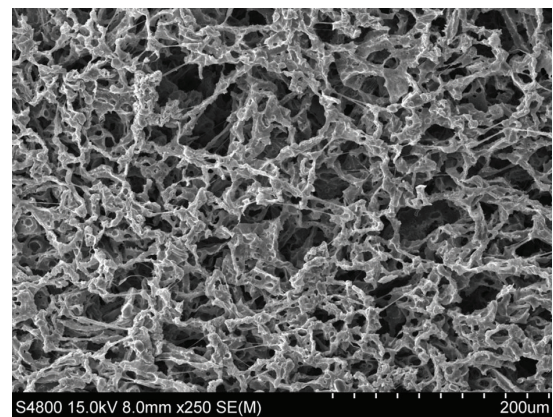

(e)

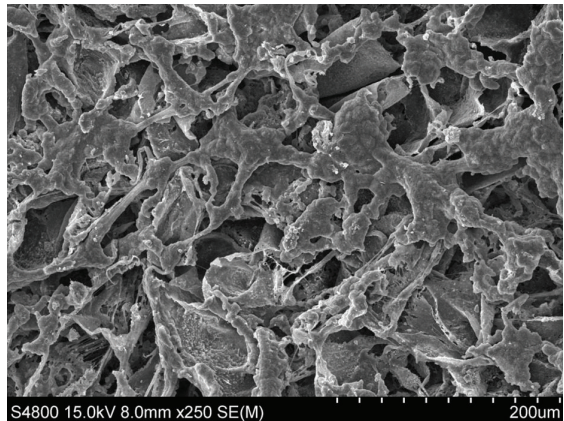

(h)

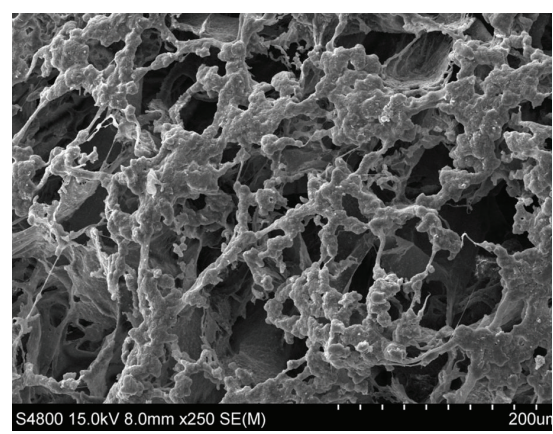

(k)

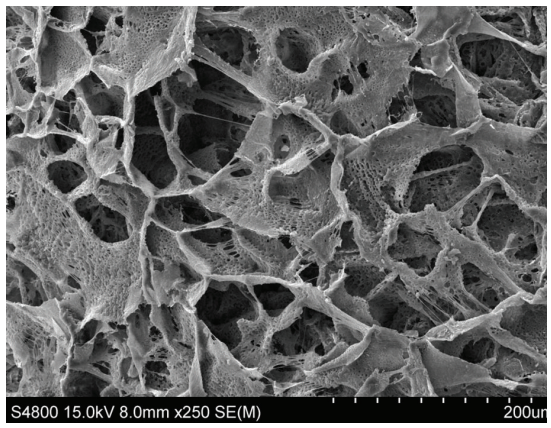

(c)

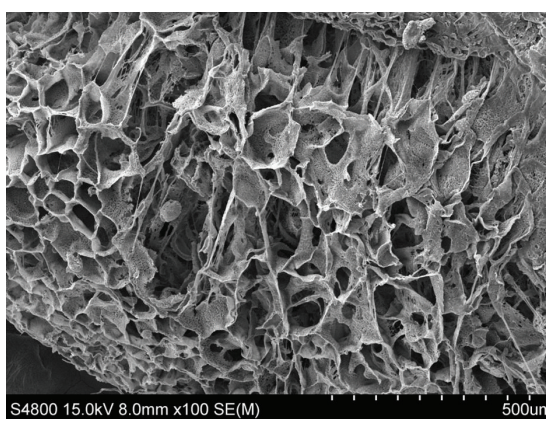

(f)

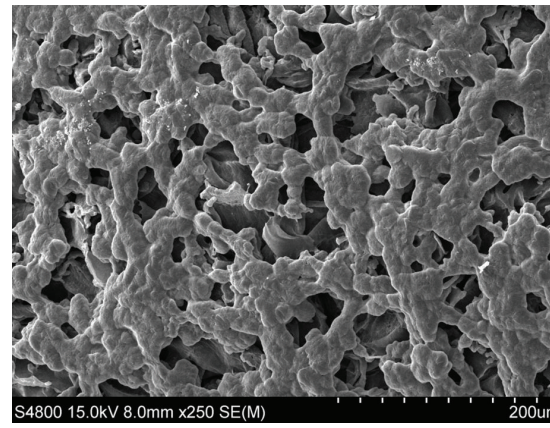

(i)

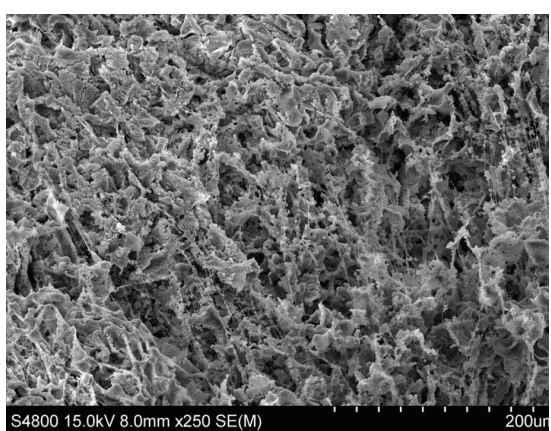

(1)

FIGURE 1: SEM observation of surface morphology of PCL $\times 250$. (a) PCL before degradation. (b) PCL/10 wt $\%$ nHA before degradation. (c) $\mathrm{PCL} / 30 \mathrm{wt} \% \mathrm{nHA}$ before degradation. (d) PCL/50 wt\% nHA before degradation. (e) PCL after degradation in vitro for $8 \mathrm{weeks}$. (f) PCL/10 wt\% nHA after degradation in vitro for 8 weeks. (g) PCL $/ 30 \mathrm{wt} \% \mathrm{nHA}$ after degradation in vitro for 8 weeks. (h) PCL/50 wt $\% \mathrm{nHA}$ after degradation in vitro for 8 weeks. (i) PCL after degradation in vitro for 16 weeks. (j) PCL/10 wt\% nHA after degradation in vitro for 16 weeks. (k) PCL/30 wt\% nHA after degradation in vitro for 16 weeks. (l) PCL/50 wt $\%$ nHA after degradation in vitro for 16 weeks. 


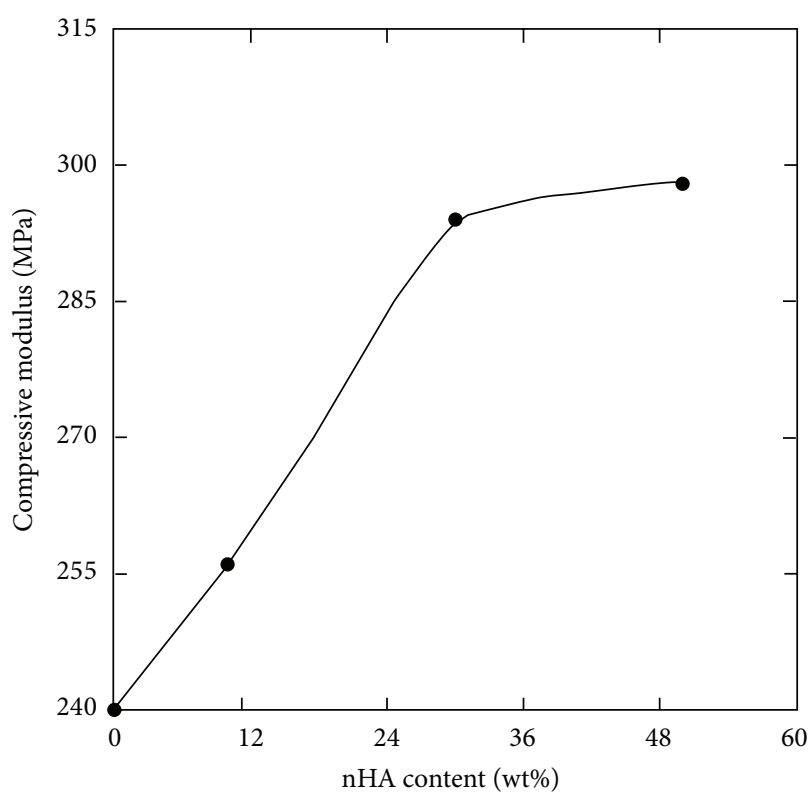

(a)

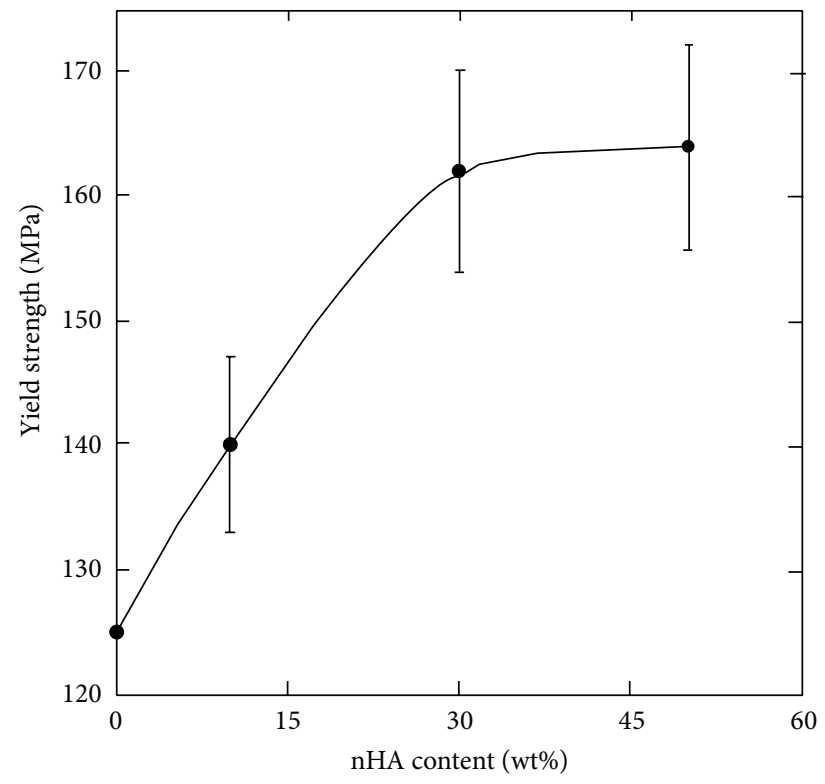

(b)

Figure 2: (a) Compressive modulus and (b) yield strength of PCL/nHA composite scaffolds as a function of nHA content (wt\%).

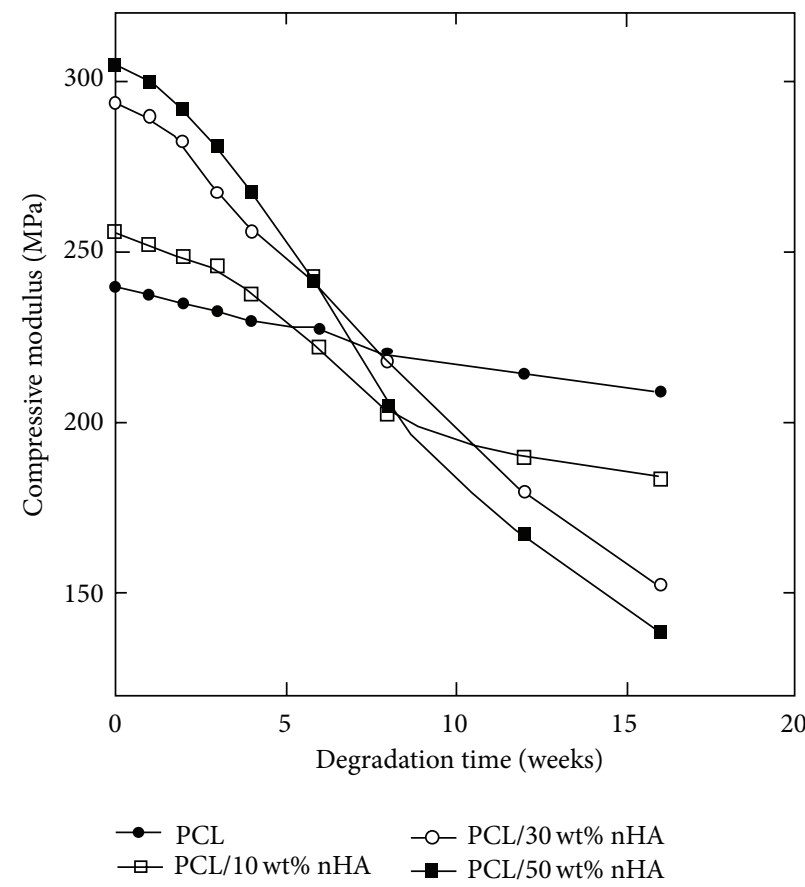

(a)

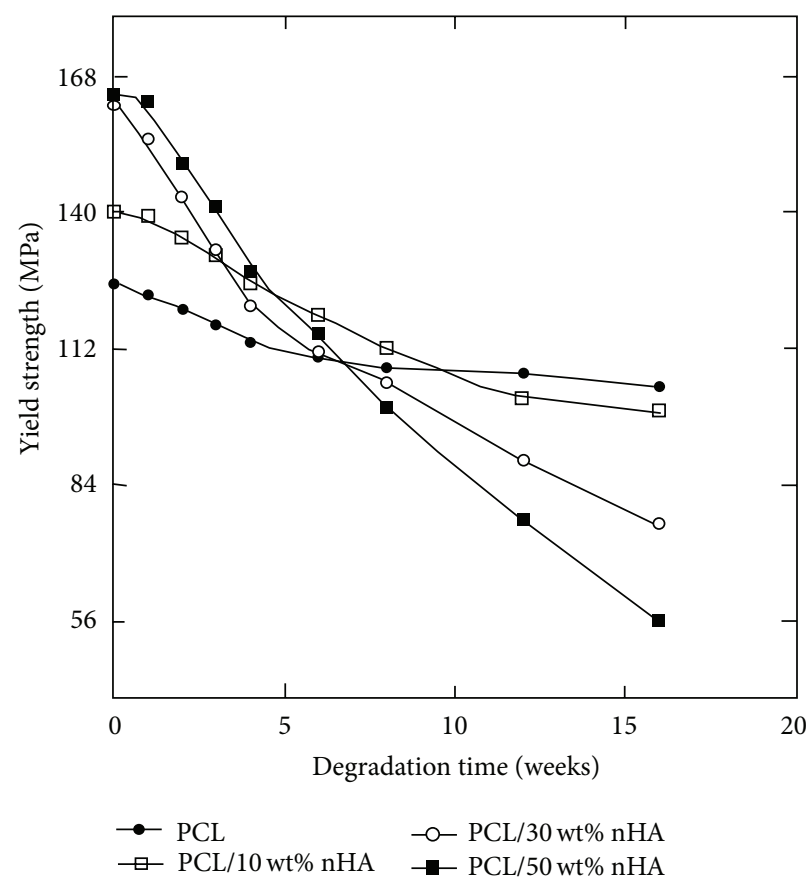

(b)

FIGURE 3: (a) Compressive modulus and (b) yield strength of PCL/nHA composite scaffolds against degradation times.

Changes in the process of in vitro degradation suppose an alteration in the structure which implies the degradation of the polymeric chain and, therefore, a decrease in the molecular weight of the scaffolds over immersion time in PBS, as can be detected in Figure 5. We can see rupture of the polymer chains is very small throughout week 12 , where there is very little appreciable variation in the molecular weight. However at the end of week 16, the molecular weight of PCL has been reduced by $30 \%$ and 39,43 , and $49 \%$ for samples with 10,30 , and $50 \%$ wt of nHA. However, the polydispersity index for all samples remained almost unchanged, with values of between 1.37 and 1.61 over the degradation period under 


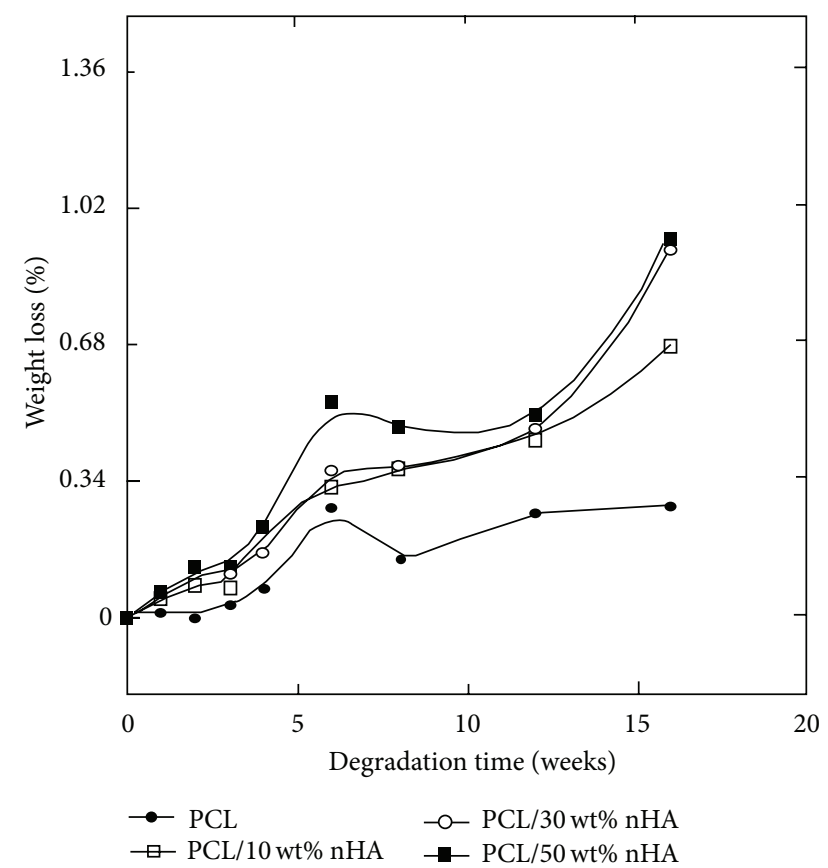

FIGURE 4: Weight loss of PCL and PCL/nHA composite scaffolds against degradation time.

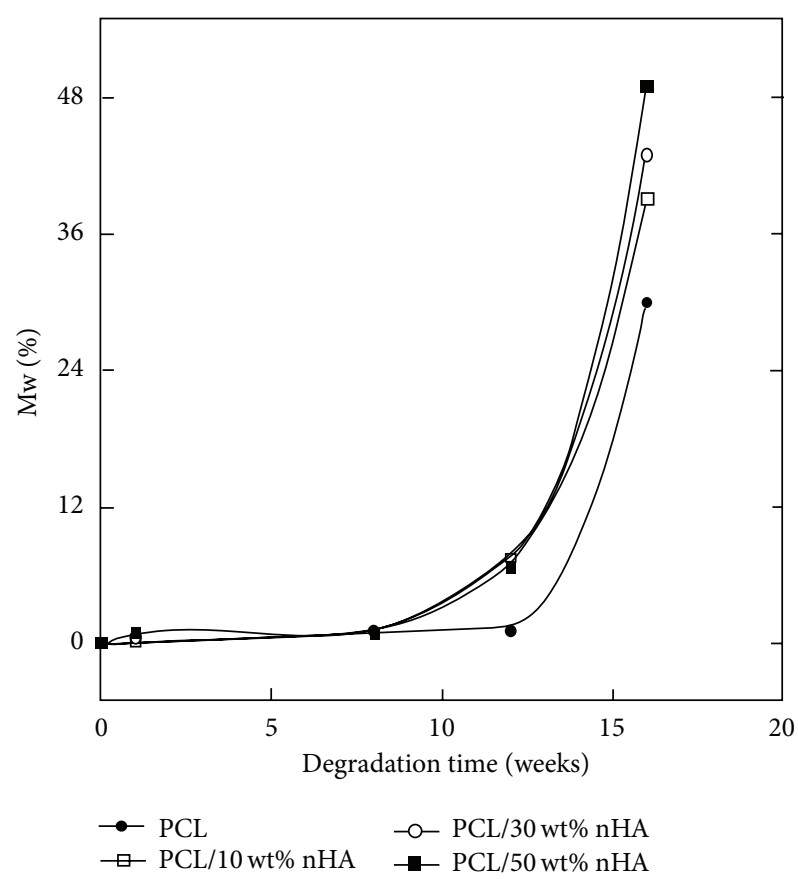

FIGURE 5: Variation of molecular weight percentage as a function of immersion time for PCL and PCL/nHA composite scaffolds.

study, and no evidence was detected of internal catalysis evidenced by uniform molecular weight distribution over time and cross-sectional examination.

This behavior suggests that the nHA particles act as an accelerator of the degradation rate in PBS. Similar results

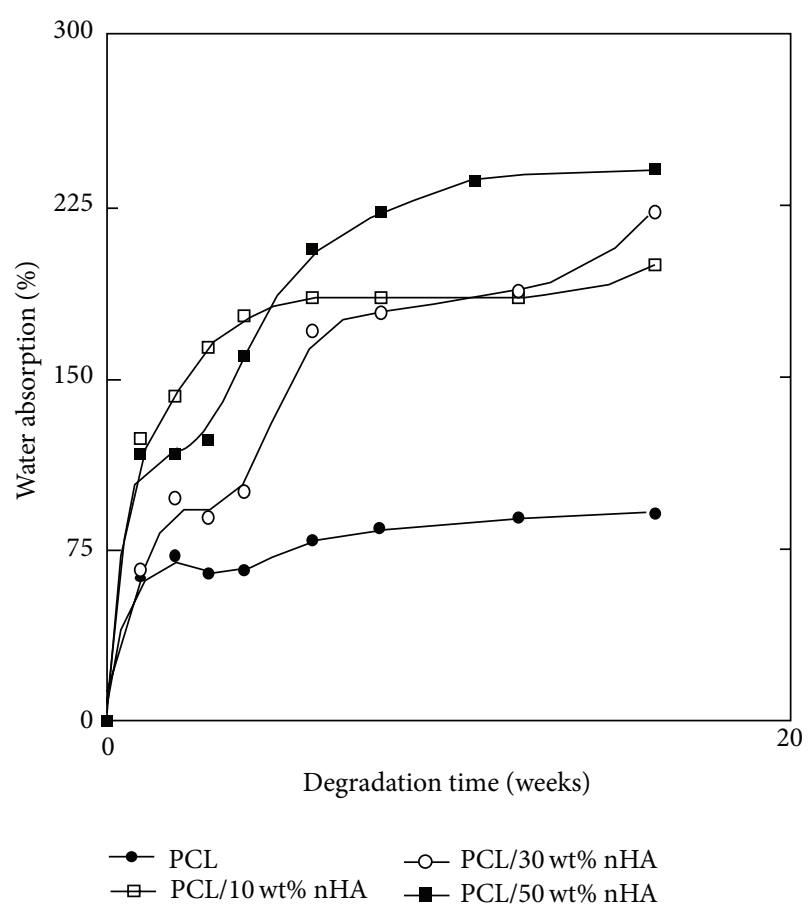

FIGURE 6: Water absorption, for PCL and PCL/nHA composite scaffolds, as a function of degradation time.

were obtained in studies with other polymers such as PLLA and copolymers such as PLGA $[11,12,14]$.

Other authors $[9,10]$ in studies of PCL scaffolds/nHA produced by melt-molding/porogen leaching technique have obtained greater weight loss but very small variations in molecular weight. This may be because the manufacturing method affects the degradation mechanism. In our case the important loss of molecular weight degradation indicates a block degradation against degradation by erosion.

3.4. Water Absorption. Figure 6 shows the \% water absorption as a function of degradation time. These water absorption curves show that the introduction of bioactive particles in the polymer scaffolds increased their capacity to absorb water during the initial incubation period. This period varies depending on the content of nHA. The maximum water absorption rate took place until the sixth week of degradation. PCL reached water absorption equilibrium, although this could not be observed in the other samples. These changes of water absorption were the result of the balance between the dissolution of oligomers in the solution and the water uptake of the residual material; in other words, the speed of absorption is reduced as a result of the dissolution of degradation products.

3.5. $p H$. Figure 7 shows the $\mathrm{pH}$ changes of the PCL and $\mathrm{PCL} / \mathrm{nHA}$ composite scaffolds as a function of in vitro degradation time. The change in $\mathrm{pH}$ of the aqueous media with degradation time was determined to check for the release of acid residues from the PLLA samples. This determination also provided information on total acid production [15-17]. 


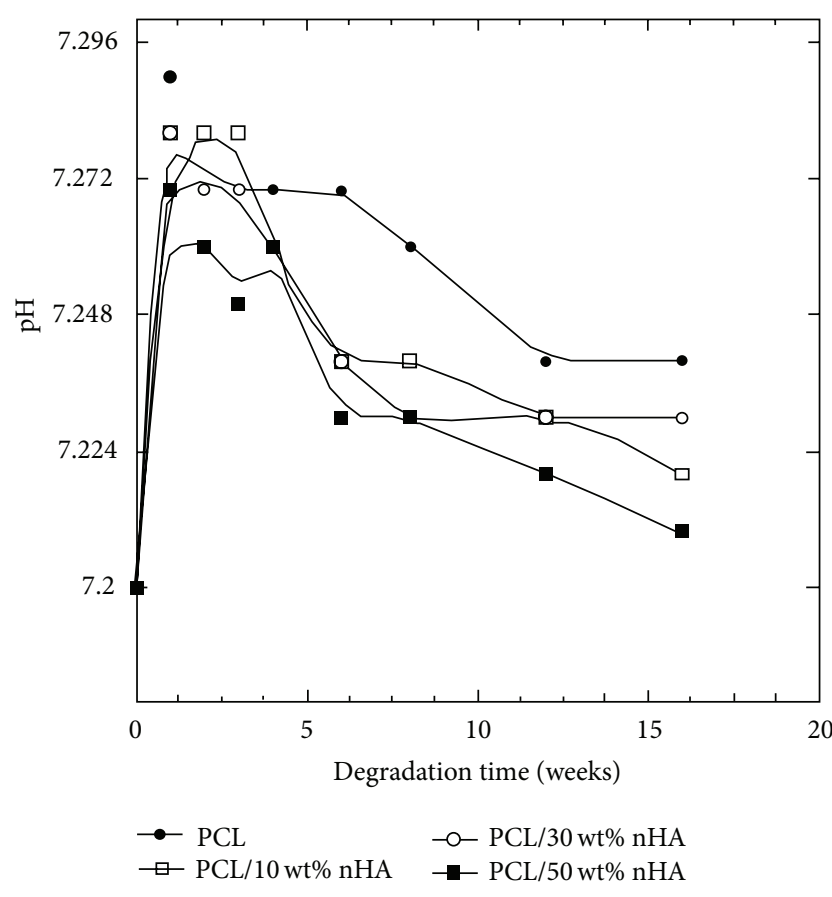

FIGURE 7: pH change of phosphate buffer solution of PCL and $\mathrm{PCL} / \mathrm{nHA}$ composite scaffolds against degradation time.

The $\mathrm{pH}$ takes its highest values in the first week of degradation and it is higher when the content of $\mathrm{nHA}$ is lower. A linear trend was observed from the sixth week for all the samples that tends to decrease the initial $\mathrm{pH}$ values. This behavior is seen most sharply in samples with a higher content of nHA.

The $\mathrm{pH}$ of the PCL and PCL/nHA $0.25 \%$ wt samples stabilized after week 10. The incorporation of most nHA particles in the scaffolds caused a decrease in the $\mathrm{pH}$ of the medium, over the degradation time, as can be seen in Figure 7. Other researchers $[15,18]$ found that the addition of bioactive particles compensated the acidification of the PBS due to acidic products of the polymer degradation, but in this case these particles had the opposite effect and accelerated the degradation rate. In the case of bulk degradation, the internal concentration of autocatalysis products produced an acidic gradient as the newly generated carboxyl end group formed during ester bond cleavage accumulated. This in turn accelerated the internal as opposed to the surface degradation, leaving an outer skin of higher molecular weight and a degraded interior with a lower molecular weight. We did not reach this level of degradation with our scaffolds, although as seen above, the polydispersity index remained largely unchanged as reported in the bioresorbable device literature $[2,15-20]$.

\section{Conclusions}

Through the study of in vitro degradation of $\mathrm{PCL} / \mathrm{nHA}$ composites, we have confirmed degradation behavior based on hydrolytic cleavage of ester groups causing random chain scission. No evidence of internal catalysis was detected in the polydispersity index values, over the degradation time. Pure PCL retained its mechanical properties, but the $\mathrm{PCL} / \mathrm{nHA}$ composites decreased as the degradation process continued, the most drastically affected samples having high nHA concentrations. The addition of nHA particles accelerated the degradation of PCL, which suggests the possibility of modulating the degradation rate of composite scaffolds by using different nanoparticle concentrations.

\section{Conflict of Interests}

The authors declare that there is no conflict of interests regarding the publication of this paper.

\section{Acknowledgment}

The technical support offered by employees at SGIker (UPV/EHU, MICINN, GV/EJ, ERDF, and ESF) is gratefully acknowledged.

\section{References}

[1] E. Díaz, I. Ibañez, and I. Puerto, Encyclopedia of Biomedical Polymers and Polymeric Biomaterials, Biopolymer Scaffolds: Manufacture, Taylor \& Francis, 2014.

[2] M. A. Woodruff and D. W. Hutmacher, "The return of a forgotten polymer-polycaprolactone in the 21st century," Progress in Polymer Science, vol. 35, no. 10, pp. 1217-1256, 2010.

[3] D.-K. Kweon, S.-B. Song, and Y.-Y. Park, "Preparation of watersoluble chitosan/heparin complex and its application as wound healing accelerator," Biomaterials, vol. 24, no. 9, pp. 1595-1601, 2003.

[4] K. F. Leong, F. E. Wiria, C. K. Chua, and S. H. Li, "Characterization of a poly- $\varepsilon$-caprolactone polymeric drug delivery device built by selective laser sintering," Bio-Medical Materials and Engineering, vol. 17, article 1477, no. 3, 2007.

[5] J. An, C. K. Chua, K. F. Leong, C.-H. Chen, and J.-P. Chen, "Solvent-free fabrication of three dimensionally aligned polycaprolactone microfibers for engineering of anisotropic tissues," Biomedical Microdevices, vol. 14, no. 5, pp. 863-872, 2012.

[6] F. E. Wiria, K. F. Leong, C. K. Chua, and Y. Liu, "Poly$\varepsilon$-caprolactone/hydroxyapatite for tissue engineering scaffold fabrication via selective laser sintering," Acta Biomaterialia, vol. 3, no. 1, pp. 1-12, 2007.

[7] K. C. Ang, K. F. Leong, C. K. Chua, and M. Chandrasekaran, "Compressive properties and degradability of poly( $\varepsilon$-caprolatone)/hydroxyapatite composites under accelerated hydrolytic degradation," Journal of Biomedical Materials Research-Part A, vol. 80, no. 3, pp. 655-660, 2007.

[8] L. Liu, Y. Wang, S. Guo, Z. Wang, and W. Wang, "Porous polycaprolactone/nanohydroxyapatite tissue engineering scaffolds fabricated by combining $\mathrm{NaCl}$ and PEG as co-porogens: structure, property, and chondrocyte-scaffold interaction in vitro," Journal of Biomedical Materials Research. Part B, Applied Biomaterials, vol. 100, no. 4, pp. 956-966, 2012.

[9] Y. Wang, L. Liu, and S. Guo, "Characterization of biodegradable and cytocompatible nano-hydroxyapatite/polycaprolactone porous scaffolds in degradation in vitro," Polymer Degradation and Stability, vol. 95, no. 2, pp. 207-213, 2010. 
[10] J. Peña, T. Corrales, I. Izquierdo-Barba, A. L. Doadrio, and M. Vallet-Regí, "Long term degradation of poly( $\varepsilon$-caprolactone) films in biologically related fluids," Polymer Degradation and Stability, vol. 91, no. 7, pp. 1424-1432, 2006.

[11] E. Díaz, I. Puerto, I. Sandonis, and I. Ibañez, "Morphology and mechanical properties of PLLA and PCL scaffolds," PolymerPlastics Technology and Engineering, vol. 53, no. 2, pp. 150-155, 2014.

[12] E. Díaz, I. Sandonis, I. Puerto, and I. Ibáñez, "In vitro degradation of PLLA/nHA composite scaffolds," Polymer Engineering and Science, 2014.

[13] C. G. Pitt, F. I. Chasalow, Y. M. Hibionada, D. M. Klimas, and A. Schindler, "Aliphatic polyesters I.: the degradation of poly(caprolactone) in vivo," Journal of Applied Polymer Science, vol. 26, no. 11, pp. 3779-3787, 1981.

[14] E. Díaz, I. Puerto, I. Sandonis, and I. Ibañez, "The effects of bioactive nanoparticles on the degradation of DLGA," International Journal of Polymeric Materials, vol. 64, no. 1, pp. 38-46, 2015.

[15] S. A. Casarin, S. M. Malmonge, M. Kobayashi, and J. A. Marcondes Agnelli, "Study on in vitro degradation of bioabsorbable polymers poly(hydroxybutyrate-co-valerate)-(PHBV) and poly(caprolactone)-(PCL)," Journal of Biomaterials and Nanobiotechnology, vol. 2, p. 207, 2011.

[16] P. Taddei, A. Tinti, M. Reggiani, and C. Fagnano, "In vitro mineralization of bioresorbable poly( $\varepsilon$-caprolactone)/apatite composites for bone tissue engineering: a vibrational and thermal investigation," Journal of Molecular Structure, vol. 744747, pp. 135-143, 2005.

[17] H. Li and J. Chang, "pH-compensation effect of bioactive inorganic fillers on the degradation of PLGA," Composites Science and Technology, vol. 65, no. 14, pp. 2226-2232, 2005.

[18] J. E. Bergsma, W. C. de Bruijn, F. R. Rozema, R. R. M. Bos, and G. Boering, "Late degradation tissue response to poly(L-lactide) bone plates and screws," Biomaterials, vol. 16, no. 1, pp. 25-31, 1995.

[19] X. L. Wang, F. Y. Huang, Y. Zhou, and Y. Wang, "Nonisothermal crystallization kinetics of poly( $\epsilon$-caprolactone)/ montmorillonite nanocomposites," Journal of Macromolecular Science, Part B: Physics, vol. 48, no. 4, pp. 710-722, 2009.

[20] I. Grizzi, H. Garreau, S. Li, and M. Vert, "Hydrolytic degradation of devices based on poly (lactic acid) size dependence," Biomaterials, vol. 16, no. 4, pp. 305-311, 1995. 

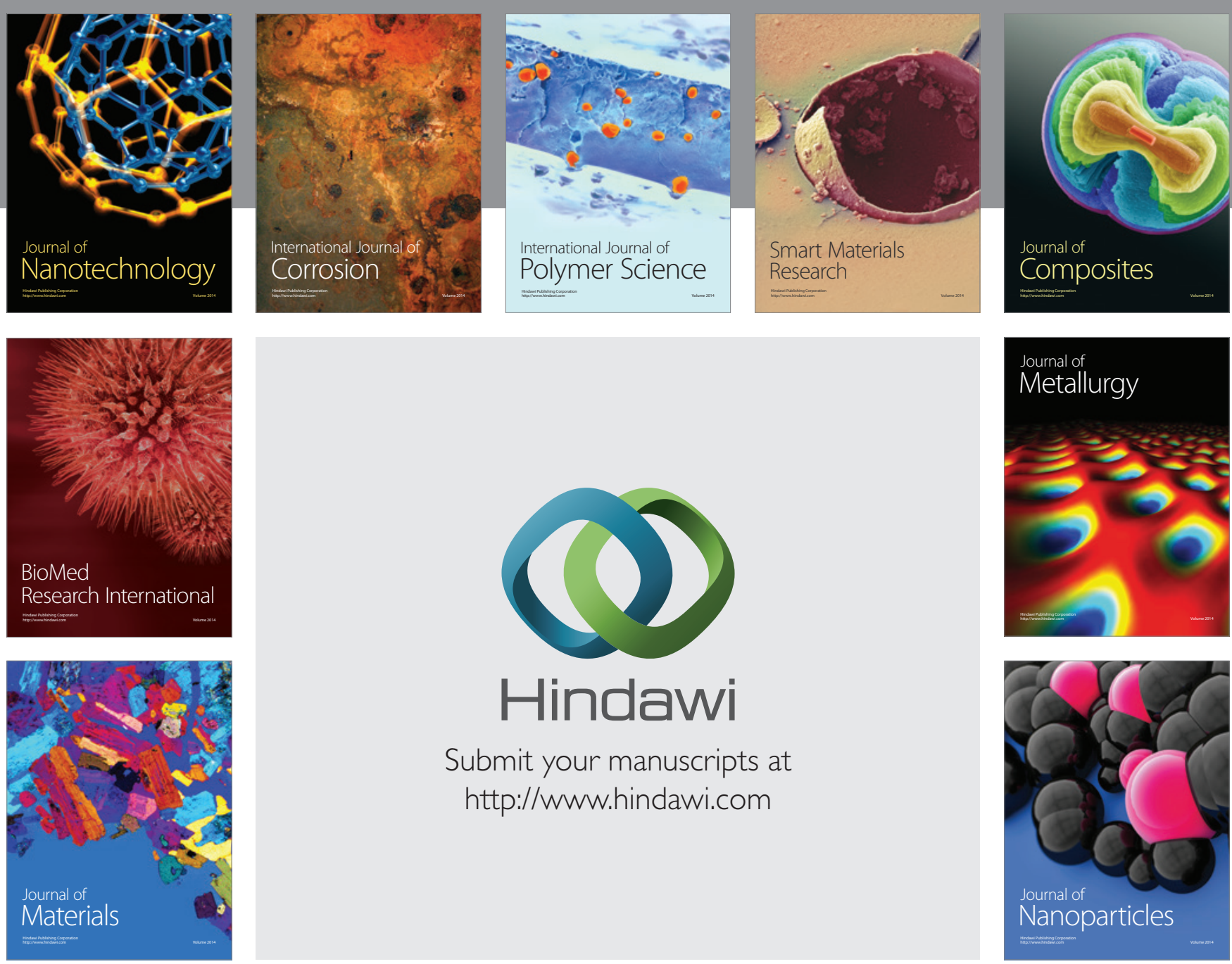

Submit your manuscripts at http://www.hindawi.com
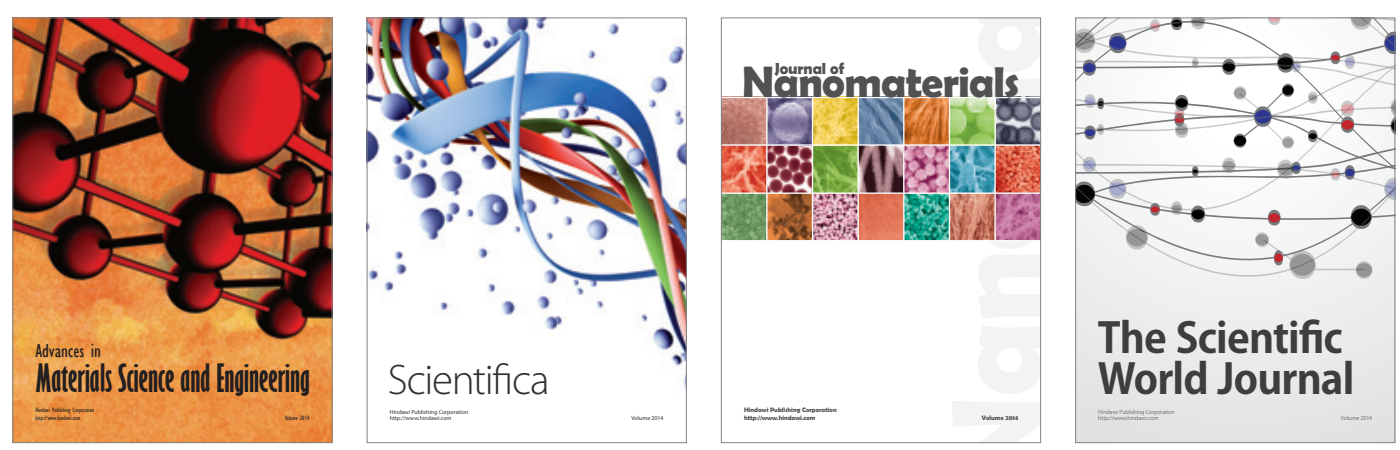

\section{The Scientific World Journal}
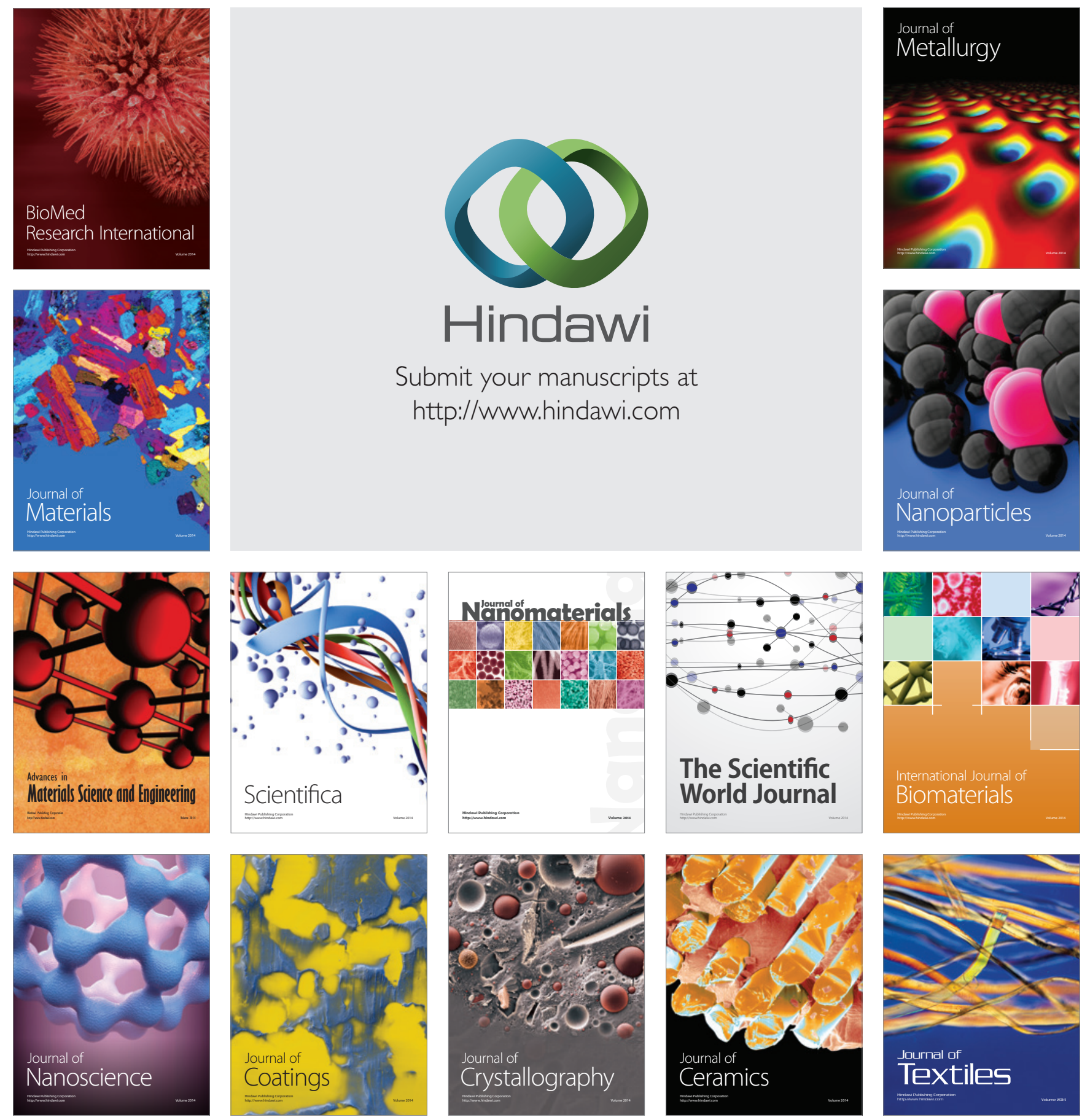